\title{
Облачный компьютинг для снижения потребления энергии в холодильных системах
}

\author{
М. А. Петренко ${ }^{1}$ С. В. Артеменко ${ }^{2}$ \\ ${ }^{1}$ Украинский научно исследовательский институт бытового машиностроения, \\ ул. Школьная 117, г. Краматорск, Украина \\ 2 Одесская национальная академия пищевых технологий, ул. Канатная, 112, г. Одесса, 65039, Украина
}

\begin{abstract}
Эволюиия энергетических систем в сторону парадигмы интеллектуальных сетей производства и распределения электроэнергии во многом определяется развитием новых технологий и их приложений. $B$ статье рассматривается подход, который использует достижения информационных технологий (Information Technology) и технологии данных (Data Technology) для управления производством, передачей и распределением энергии. Рассмотрена интеллектуальная инфраструктура, которая направлена на управление сетями электроснабжения, включающими в качестве потребителей энергии, холодильнье системы. Снижение потребления энергии в холодильных системах базируется на применении облачного компьютинга, который предоставляет необходимые интерфейсы и набор услуг для взаимодействия с интеллектуальными счетчиками и автоматизации системы распределения. Для сжатия большого объема данных, которые генерируются в результате мониторинга показателей работь холодильного прибора, используется «вейвлет» преобразование сигналов. Дана оценка снижения потребления энергии в холодильной системе за счет технологии облачного компьютинга.
\end{abstract}

Ключевые слова: Холодильные системы; Энергоэффективность; Облачный компьютинг; Интеллектуальные сети электроснабжения

\section{Хмарний комп'ютинг для зниження споживання енергії в холодильних системах}

\section{М. О. Петренко С. В. Артеменко ${ }^{2}$}

${ }^{1}$ Український науково-дослідний інститут побутового машинобудування, вул. Шкільна, 117, м. Краматорськ, Україна

2 Одеська національна академія харчових технологій, вул. Канатна, 112, м. Одеса, 65039, Україна

\begin{abstract}
Еволюиія енергетичних систем у бік парадигми інтелектуальних мереж виробництва та розподілу електроенергї багато в чому визначається розвитком нових технологій та їх застосувань. B статті розглядається підхід, який використовує досягнення інформаиійних технологій (Information Technology) ma технологій даних (Data Technology) для управління виробництвом, передачею та розподілом енергії. Розглядається інтелектуальна інфраструктура, яка спрямована на управління мережами електропостачання, щз включають в якості споживачів енергї, холодильні системи. Зниження споживання енергї в холодильних системах трунтується на використанні хмарного комп'ютинга, який надає необхідні інтерфейси та набір послуг для взаємодї з інтелектуальними датчиками та автоматизації системи розподілу. Для стиснення великого обсягу даних, які генеруються в результаті моніторингу показників роботи холодильного приладу, використовується «вейвлет» - перетворення сигналів. Дана очінка зниження споживання енергії в холодильній системі за рахунок технологї хмарного комп'ютинга.
\end{abstract}

Ключові слова: Холодильні системи; Енергоефективність; Хмарний компюттиг; Інтелектуальні мережі електропостачання 


\section{1 Введение}

Использование электроэнергии бытовыми холодильными системами составляет около $30 \%$ месячного потребления энергии. Данное обстоятельство приводит к увеличению вклада холодильной техники в глобальное потепление и эмиссию парниковых газов в окружающую среду. Производство электроэнергии на основе ископаемого топлива отвечает за почти половину выбросов $\mathrm{CO}_{2}$ и с каждым годом становится все дороже. Наиболее распространенная точка зрения на получение экологически безопасных технологий производства электроэнергии - это переход к возобновляемой энергетике, использующей солнечную энергию, энергию ветра, геотермальную энергию и др. Иной подход, который развивается наиболее интенсивно в последнее время, заключается в подключении информационных технологий (Information Technology) и технологии данных (Data Technology) для управления производством, транспортом и распределением энергии. Одним из перспективных направлений указанного подхода является создание интеллектуальных электрических сетей (Smart Grid). B данной статье концепция интеллектуальных сетей рассмотрена как синергетическая комбинация традиционных электрических сетей с потребителем энергии (в нашем случае, холодильных систем) в сочетании $\mathrm{c}$ коммуникационной инфраструктурой. Характерной чертой таких сетей являются большие объемы данных (Big Data), обработка которых требует современных стратегий в управлении различными процессами.

Здесь мы обсуждаем модель облачного компьютинга (Cloud Computing) для принятия решений в приложениях Smart Grid. Модель облачного компьютинга представляет собой сервисное обслуживание через Интернет потребителей информации для решения крупномасштабных задач энергетического менеджмента и управления ресурсами спроса. Облачные технологии [1-7] обеспечивают снижение затрат на программное обеспечение, увеличение объёма памяти и скорости вычислений, что привело к массовому внедрению модели облачных вычислений в технологии Smart Grid [8-14]. Создание «интеллектуальных сетей» в странах ЕС опирается на «Платформу европейских умных сетей электроснабжения» (Smart Grid European Technology Platform) [15]. Развитие технологии Smart Grid означает фундаментальную реорганизацию рынка услуг электроэнергетики, хотя терминология предполагает только технические аспекты развития сетевой инфраструктуры. Подробный анализ литературы, посвященной интеллектуальным инфраструктурным системам, интеллектуальным системам управления и интеллектуальным системам защиты, приведен в [11].

Цель данной работы заключается в разработке подхода к снижению энергопотребления в холодильных системах, входящих в состав интеллектуальных электрических сетей, в реальных условиях эксплуатации за счет использования методов облачного компьютинга.
Вначале мы рассматриваем модель виртуальной энергетической системы, в состав которой включена модель виртуальной холодильной системы. Следующий раздел посвящен описанию архитектуры облачного компьютинга. Для сжатия большого объема данных, которые генерируются в результате мониторинга показателей работы холодильного прибора в рамках виртуальной энергетической системы, используется «вейвлет» - преобразование сигналов. Последнее обобщает методы спектрального анализа временных функций на основе семейства функций, которые локализованы по частоте и времени. Далее обсуждаются результаты, иллюстрирующие повышение энергоэффективности холодильных систем за счет интеллектуального управления ресурсами всей энергетической системы. Наконец, в заключение обсуждаются перспективы применения облачного компьютинга в холодильной технике.

\section{2 Интеллектуальные электрические сети}

Облачный компьютинг играет ключевую роль в создании будущих интеллектуальных сетей в качестве новой технологии, обеспечивающей удобный сетевой доступ по запросам вычислительных алгоритмов управления поведением потребителей энергии, которые требуют надежного, устойчивого и экономичного электроснабжения.

Любая интеллектуальная сетевая инфраструктура должна поддерживать двустороннюю связь между производителями и потребителями энергии в режиме реального времени и позволять системам программного обеспечения как на стороне производителя, так и потребителя контролировать и управлять процессами передачи энергии [16].

Моделирование сложных энергетических систем целесообразно осуществить при помощи виртуальных компьютерных мультиагентных моделей $\{\mathrm{MAS}\}$, которые имитируют взаимодействие автономных подсистем (агентов) между собой и окружающей средой с целью интегральной оценки эффективности системы. Структура виртуальной энергетической системы, ранее представленная в работе [17], является связующим звеном между различными типами тепло- и электрогенерирующих мощностей, что позволяет устранить противоречие между требованиями рынка и возможностями технической реализации. Системы MAS относятся к числу так называемых «самоорганизующихся систем», которые находят наилучшее решение без постороннего вмешательства.

На рисунке 1 приведена возможная архитектура облачного компьютинга, включающая свойства и процессы изменения состояния (услуги). Термин свойства (состояния) обозначает наличие структурированных и неструктурированных данных огромных объёмов, которые обрабатываются программными средствами, представляющими альтернативу стандартным методам управления базами данных. Появление больших данных в инженерных приложениях чаще всего результат непрерывного мониторинга различных показателей работы электрических сетей. 
Процесс изменения состояния отражает взаимодействие пользователей и потребителей энергии при помощи облачного компьютинга. Реализация облачных вычислений базируется на трех основных видах услуг:

- модель обслуживания (МО), которая обеспечивает доступ пользователя к необходимому программному обеспечению при помощи виртуальных машин;
- виртуальные машины (BM) [18], которые предоставляют непосредственный доступ через облако к требуемым моделям без загрузки и установки необходимого программного обеспечения;

- программное обеспечение (ПО), которое поддерживает все вычисления в облачной среде через доступ к веб-браузерам, минуя их установку на собственном компьютере.

СВОЙствА

услуги

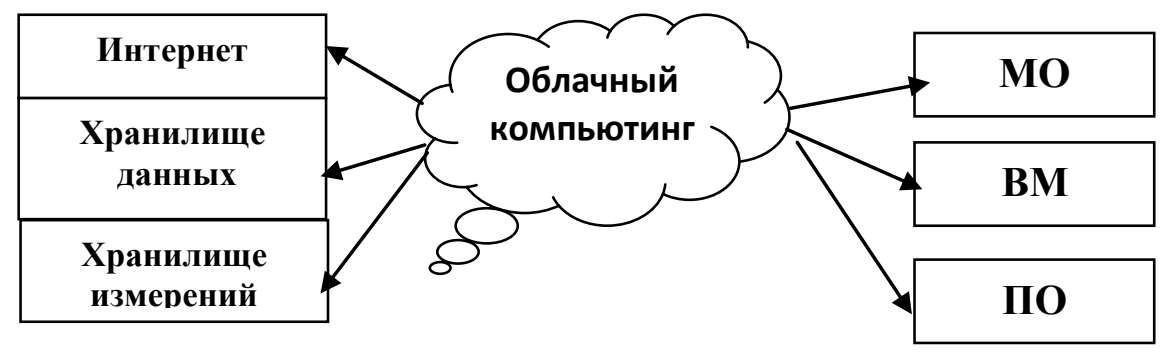

Рисунок 1 - Архитектура облачного компьютинга

Облачный компьютинг - это новая вычислительная модель, которая содержит общие ресурсы в рамках хранилища данных в Интернете и вычислительных алгоритмов как поставщика услуг.

\section{3 Сжатие результатов мониторинга показателй холодильной установки}

Методы функциональных испытаний бытовых холодильных установок и требования к испытательному оборудованию регулируются ISO 8187 (EN 28187) «Бытовые холодильные устройства Холодильники-морозильники - характеристики и методы испытаний» (Household refrigerating appliancesRefrigerator-freezers - characteristics and test methods). Выполнение указанных стандартов требует следующих измерений, которые отражают реакцию холодильной системы на различные маркетинговые требования: потребление энергии, температура хранения продуктов, устойчивость работы прибора, температура окружающей среды. Потребление энергии бытового холодильного прибора в течение 24 часов в неизменных рабочих условиях определяют при температуре окружающей среды $25^{\circ} \mathrm{C}$ для климатических классов SN, N, ST. Для повышения качества температурных измерений был использован гибридный рекордер, измеряющий одновременно от 10 до 20 каналов. Термопары измеряли входные данные, которые одновременно записывали и передавали на персональный компьютер с интервалом 5 сек. Температурные данные в различных точках холодильных камер, в соответствии со схемой на рисунке 2, при незагруженном/загруженном пространстве холодильной камеры приведены на рисунках 3 и 4.
Протоколы испытаний содержат следующую информацию об испытаниях и приведены в форме EXCEL-файлов в хранилище измерений. Они включают сведения о температуре окружающего воздуха, величине температуры отделения для свежих продуктов $T \mathrm{~m}$ и величины $T_{1}, T_{2}, T_{3}$, величине температур отделений для охлаждения и замораживания продуктов.

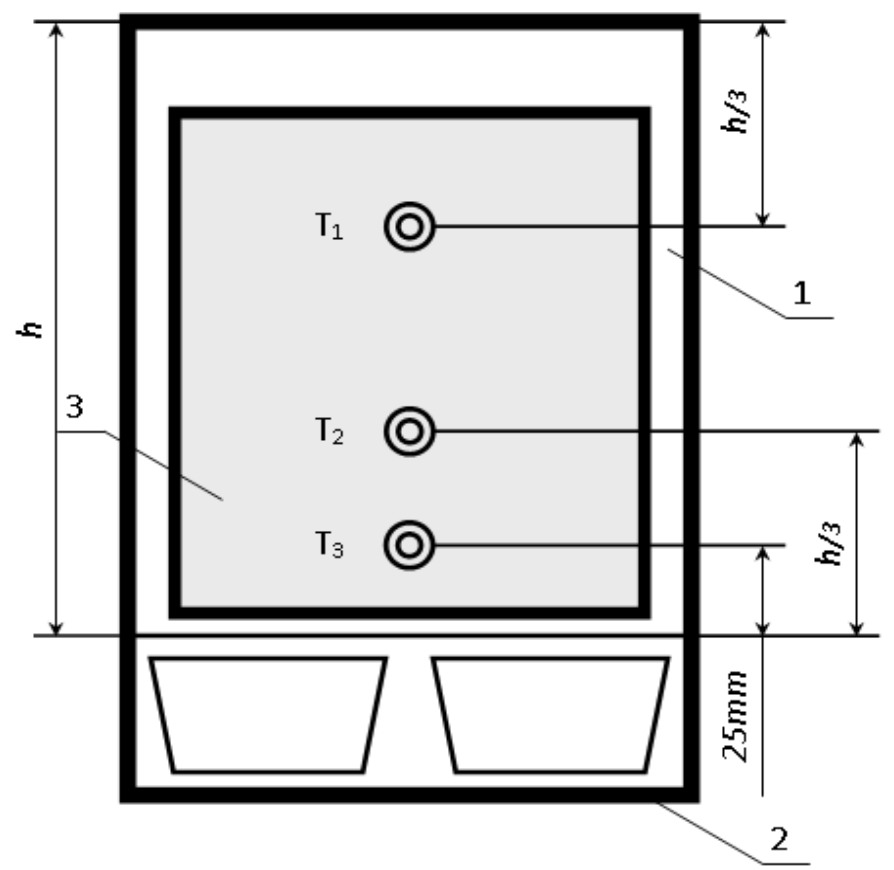

1 - отделение для свежих продуктов; 2 -отделение для охлажденных продуктов; 3 -испаритель; $h$ - высота отделения для свежих продуктов

Рисунок 2 - Схема расположения датчиков температуры 


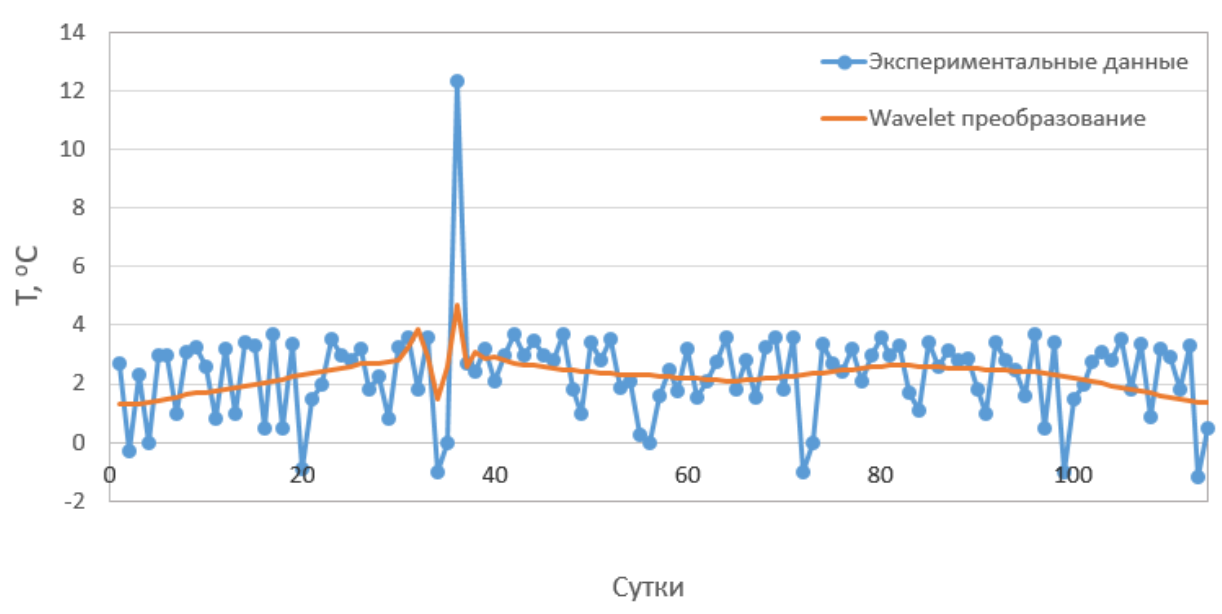

Рисунок 3 - Изменение температуры в нижней части незагруженной холодильной камеры

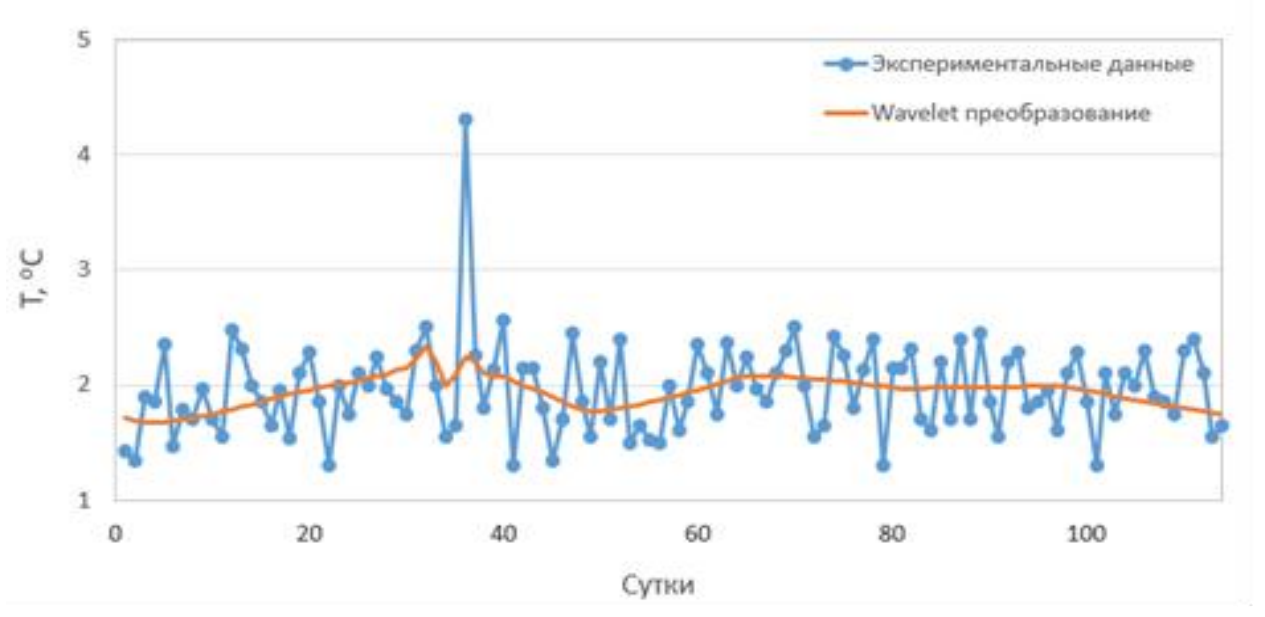

Рисунок 4 - Изменение температуры в верхней части загруженной холодильной камеры

Показатели непрерывной работы холодильной установки передаются и содержатся в хранилище измерений, в котором находится большой объем данных. Для сжатия значительного объема данных, которые генерируются в результате мониторинга показателей работы холодильного прибора в рамках виртуальной энергетической системы, в работе использовано вейвлет преобразование сигналов. Последнее обобщает методы спектрального анализа временных функций на основе семейства функций, которые локализованы по частоте и времени [19]. На рисунках 3 и 4 показаны результаты вейвлет преобразования измерений температуры в различных частях холодильной камеры. Расчеты проводили на базе Wavelet Toolbox Matlab [20]. Преимуществом данного преобразования является возможность проводить операции свертки, сглаживания, фильтрования и т.п. без потери существенных деталей. Возможные особенности поведения измеряемых показателей из-за сбоя оборудования, различных шумов и других причин легко обнаружить и устранить.

\section{4 Облачный подход к минимизации стоимости электроэнергии}

Повышение надежности сетей электроснабжения, в основном, базируется на применении резервных генераторов. Последние потребляют ископаемое топливо и, следовательно, увеличивают эмиссию углекислого газа в окружающую среду. Поэтому более перспективным является замена физической выработки энергии на виртуальную. Эта идея известна как реакция потребителя на корректировку кривых «спрос-предложение» [21], [22]. На рисунке 5 показаны преимущества реакции спроса на рынках электроэнергии и рекомендации по их осуществлению, приведенные в докладе для конгресса США [21]. Наличие возобновляемых источников энергии и холодильных систем как аккумуляторов энергии в составе сетей электроснабжения позволяет использовать облачные приложения в качестве хранилища виртуальной энергии [23]. Потребители энергии, которые включены в сеть и обладают 
доступом к виртуальному хранению энергии, могут минимизировать стоимость электроэнергии и удовлетворять возможные запросы других потребителей, что повышает надёжность сети электроснабжения.

Рассмотренная инфраструктура облачного компьютинга в среде интеллектуальных сетей требует программное обеспечение, поддерживающее вычисления в реальном времени для обработки огромного количества данных. К сожалению не существует единого подхода к эффективному управлению спросом в интеллектуальных сетях. Противоречивость требований производителей и потребителей энергии делает невозможным существования единого критерия управления и должна отражать возникающую неопределенность в принятии решений.

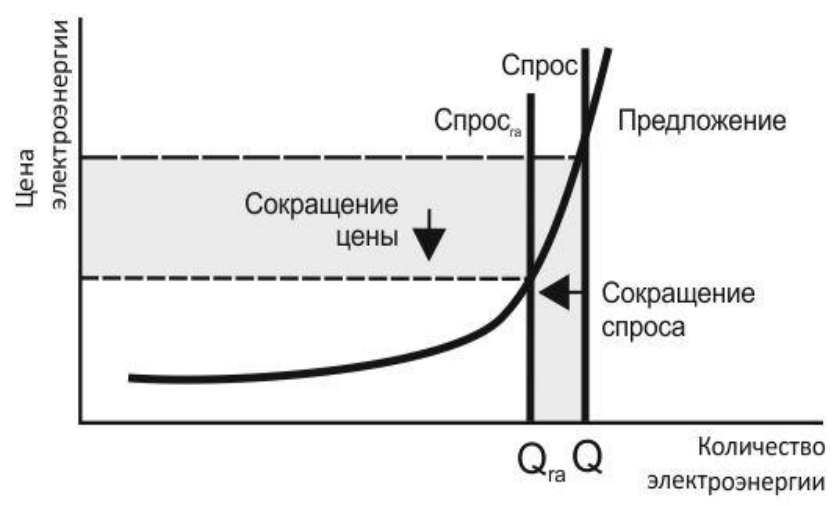

\section{Рисунок 5 - Возможная реакиия потребителя на сдвиг} кривой спрос - предложение [21]

В данной работе выбор критерия управления осуществляли на основе концепции устойчивого развития. Математическая реализация указанной концепции предполагает решение задачи оптимального управления при наличии многих критериев. Выбор критериев управления и ограничений носит субъективный характер и зависит от энергетических, экономических, социальных, психологических и политических факторов. Взаимодействие множества факторов для достижения цели следует понимать в смысле компромисса между несовместимыми критериями $K(X)$ за счет соответствующего выбора вектора стратегии управления $\boldsymbol{X}$.

Найти

$$
\text { Optimize } K\left[K_{l}(X), \ldots, K n(X)\right]
$$

при ограничениях на ресурсы

$$
C_{i} \equiv G_{L i} \leq G_{i}(X) \leq G_{U i}, i=1,2, \ldots, k
$$

и стратегии управления

$$
x_{L i} \leq x_{i} \leq x_{U i}, i=1,2, \ldots, m,
$$

где $K_{i}(X) \quad(i=1 \ldots n) \quad$ - локальные критерии эффективности виртуальной сети электроснабжения;
$X\left(x_{1}, x_{2}, \ldots, x_{m}\right)$ - вектор стратегий управления; $G_{L i}, G_{U i}$ - нижний и верхний пределы ограничений $G_{i}(X$,$) ,$ соответственно, и $x_{L i} \leq x_{i} \leq x_{U i}$ - нижняя и верхняя границы для искомых стратегий управления.

Многокритериальный подход базируется на комбинации формальной и неформальной процедур принятия решений для нахождения приемлемых стратегий управления. Правильная стратегия для рассматриваемой холодильной системы выполняется с помощью решения многокритериальной задачи (1) (3). Поиск компромиссного решения осушествляли на основе алгоритмов нечеткой термоэкономической оптимизации энергопреобразующих систем [24]. Окончательный выбор стратегии Xорt предполагает достижение компромисса между выбранными показателями эффективности и носит субъективный характер. Группа оптимизационных задач для пользователей решается с целью создания интегрированной функции при определенных ограничениях для минимизации затрат и максимизации прибыли для поставщика электроэнергии. Существует множество алгоритмов решения таких задач. В их основе лежит программа реагирования на запросы от потребителя, сервера и хранилища данных в структуре облачного компьютинга. В зависимости от выбранной стратегии пользователю удается наилучшим образом изменить нагрузку и получить преференции от производителя электроэнергии.

\section{5 Результаты}

Оценку перспектив технологии облачного компьютинга для снижения стоимости электроэнергии, потребляемой холодильной системой, проводили на основе вычислительных экспериментов. В качестве целевой функции при переходе от векторной задачи оптимизации (1)-(3) к скалярной использовали простейшую схему аддитивной свертки [24]. В этом случае минимизация энергетической и стоимостной целевых функций совпадает, поскольку в классическом термоэкономическом анализе ценовые и энергетические показатели отличаются лишь множителем преобразования $k$ [денежн. единица/кВт ч]. В общем случае такая термоэкономическая модель имеет множество недостатков и разработаны альтернативные и более адекватные подходы, учитывающие экологические и другие ограничения. На рисунке 6 показана относительная цена потребляемой электроэнергии для холодильной системы в случае стандартной ситуации (верхняя кривая) и с учетом компромиссной стратегии (нижняя кривая).

Приведенные кривые соответствуют изменению холодильного коэффициента, который преобразован в относительный стоимостный показатель. Здесь рассмотрена простейшая схема свертки локальных показателей, которая демонстрирует минимальное потребление энергии во время пиковой нагрузки (12...14 часов). Более корректная стратегия должна учитывать взаимодействие потребителя и поставщика электроэнергии в режиме реального времени. Соответствующие алгоритмы оптимизации строятся на теоретико-игровых моделях. 


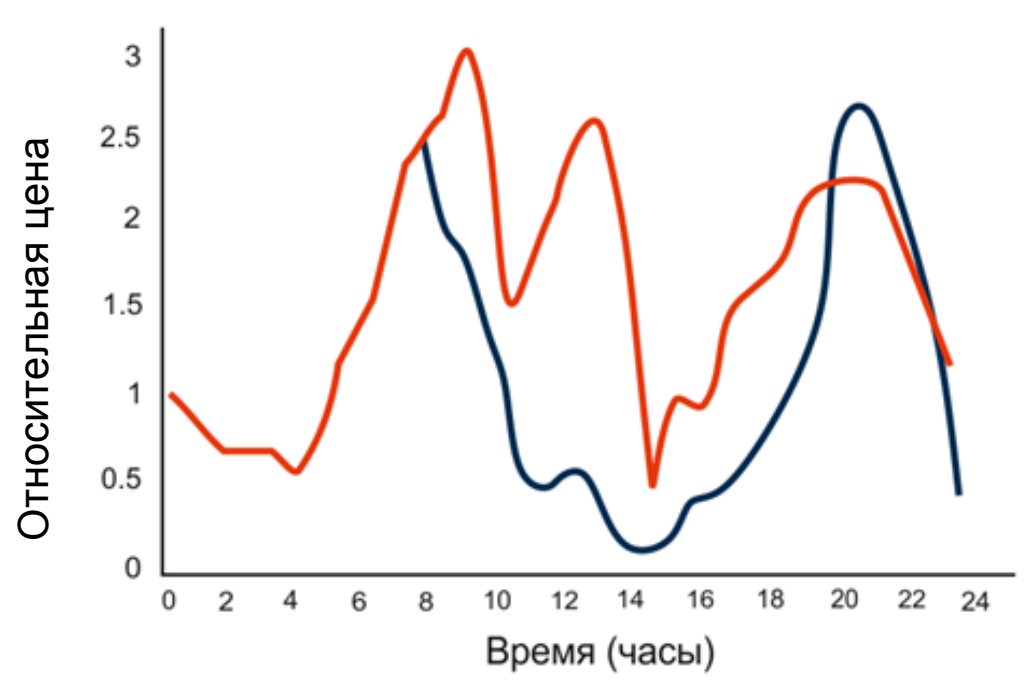

Рисунок 6 - Изменение относительной цеены потребляемой электроэнергии для холодильной системы

\section{Выводы}

Представление об умных сетях играет ключевую роль в будущих энергетических сценариях, в которых работа холодильных систем направлена на стремление обеспечить компромиссное согласование между производством и потреблением энергии. Взаимодействие потребителей энергии в рамках интеллектуальной системы электроснабжения является одним из перспективных направлений в снижении потребления энергии в системах холодильной техники. В предлагаемом подходе рассмотрена облачная схема расчетов, минимизирующих потребление энергии холодильной системы за счет изменения нагрузки.

Расчеты для интеллектуальных сетей электроснабжения (Smart Grid) могут быть выполнены с помощью сервисной модели Cloud Computing в рамках среды Matlab, ориентированной для решения стандартных многоцелевых задач оптимизации либо специально разработанных алгоритмов, специфичных для конкретных задач обработки больших объемов данных. Важными требованиями являются параллельная обработка данных и прямой доступ к ресурсам и услугам, которые желательны для технологий Smart Grid. В настоящее время модели облачного компьютинга находятся на стадии исследований и разработок и еще не относятся к числу стандартных процедур. Основные ограничения, с которыми сталкивается применение модели Cloud Computing, связаны с вопросами безопасности и надежности. Рассмотрение данных проблем является предметом дальнейших работ в этом направлении.

\section{Литература}

1. Bera S., Misra S., Rodrigues P. C. Cloud Computing Applications for Smart Grid: A Survey 2017/ https://www.researchgate.net/publication/262116090
2. Qaisar E. Introduction to cloud computing for developers: Key concepts, the players and their offerings/ 2012. Proc. of IEEE TCF Pro IT, pp. 1-6.

3. Popeanga J. Cloud Computing and Smart Grids. 2012. Database Systems Journal, vol. 3, no. 3, pp. 57-66.

4. Lakhani A. The Definition of Cloud Computing. 2011. http://www.cloudcentrics.com

5. Mell P., and Grance T. The NIST Definition of Cloud Computing, US National Institute of Science and Technology Std., 2011. [Online]. Available: http://csrc.nist.gov/publications/nistpubs/800-145/SP800-

145.pdf

6. Guo Y., Pan M., and Fang Y. Optimal Power Management of Residential Customers in the Smart Grid, 2012. IEEE Trans. on Parallel and Distributed Systems, vol. 23, no. 9, pp. 1593 - 1606.

7. Markovic D.S., et al. Smart power grid and cloud computing. 2013. Renew. Sustain. Energy Rev. 24, pp. $566-577$.

8. Nozaki Y., Tominaga T., Iwasaki N., and Takeuchi A. A technical approach to achieve smart grid advantages using energy management systems. 2011. Proc. of IEEE Intl. Conf. on WCSP, pp. 1-5.

9. Fang X., Misra S., and Yang D. Smart Grid. The New and Improved Power Grid: A Survey. 2012. IEEE Comm. Surveys \& Tutorials, vol. 14, no. 944-980.

10. Hashmi M., Hanninen S., and Maki K. Survey of smart grid concepts, architectures, and technological demonstrations worldwide. 2011. in Proc. of IEEE PES ISGT, pp. 1-7.

11. Zhou J., Hu R. Q., and. Qian Y. Scalable Distributed Communication Architectures to Support Advanced Metering Infrastructure in Smart Grid. 2012. IEEE Trans. on Parallel and Distributed Systems, vol. 23, no. 9, pp. $1632-1642$.

12. Metke A. and Ekl R. Smart Grid security technology. 2010. Proc. of IEEE Conf. on ISGT, pp. 1-7.

13. Luo F., Dong Z. Y., Chen Y., Xu Y., Meng K., and Wong K. P. Hybrid cloud computing platform: The next 
generation IT backbone for smart grid. 2012. Proc. of IEEE Conf. on PES General Meeting, pp. 1-7.

14. Metke A. and Ekl R. Smart Grid security technology. 2010. Proc. of IEEE Conf. on ISGT, pp. 1-7.

15. Smart Grids European Technology Platform 2011. www.smartgrids.eu.

16. Goulden M., Redwell B., Rennick-Egglestone S., Rodden T., Spence A. 2014. Smart grids, smart users? The role of the user in demand side management Energy Research \& Social Science 2, 21-29

17. Петренко М.А., Тришин Ф.А., Мазур В.А. Повышение энергоэффективности холодильных систем в интеллектуальных сетях электроснабжения. Холодильная техника и технология, т.53, вып. 4. 2017. - C. 42-49.

18. Prodan R. and Ostermann S. A. Survey and Taxonomy of Infrastructure as a Service and Web Hosting Cloud Providers. 2009. Proc. of Intl. Conf. on Grid Computing. 2009. pp. 1-10.

19. Астафьева Н.М. Вейвлет-анализ: основы теории и примеры применения. 1996. Успехи физических наук. 66, №11, 1146 - 1170 .

20. https://www.mathworks.com/products/wavelet.html

21 . Benefits of demand response in electricity markets and recommendations for achieving them. Report to the United States Congress, Feb. 2006. https://eetd.lbl.gov/sites/all/files/publications/report-lbnl1252d.pdf

22. Erol-Kantarci M. and Mouftah H. TOU-Aware Energy Management and Wireless Sensor Networks for Reducing Peak Load in Smart Grids. , 2010. Proc. of IEEE Conf. on VTC-Fall, pp. 1-5.

23. Nezamabadi P. and. Gharehpetian G. Electrical energy management of virtual power plants in distribution networks with renewable energy resources and energy storage systems. 2011. Proc. of Electrical Power Distribution Networks, Bandar Abbas. pp. 1-5.

24. Mazur V. Fuzzy thermoeconomic optimization of energy-transforming systems. 2007. Applied Energy, 84, p. 74.

Отримана в редакції 01.11.2017, прийнята до друку 08.12.2017

\title{
Cloud Computing for Energy Efficient Refrigeration Systems
}

\author{
M. O. Petrenko ${ }^{1}$, S. V. Artemenko ${ }^{2}$ \\ ${ }^{1}$ Ukrainian Research Institute of Household Engineering (UkrNIIbytMash). 117, Shkolnaya str., Kramatorsk, Ukraine \\ ${ }^{2}$ Odesa National Academy of Food Technologies, 112, Kanatna str., Odesa, 65039, Ukraine
}

\begin{abstract}
The evolution of energy systems towards the paradigm of intelligent networks for the production and distribution of electricity is largely determined by the development of new technologies and their applications. The article considers an approach that uses the achievements of information technology and data technology for management of production, transmission and distribution of energy. The intellectual infrastructure is considered, which is aimed at the management of power supply networks, including the refrigeration systems as energy consumers. Reducing energy consumption in refrigeration systems is based on the use of cloud computing, which provides the necessary interfaces and a set of services for interaction with smart meters and automation of the distribution system. To compress the big data that is generated as a result of monitoring the performance of the refrigeration appliance, a "wavelet" transformation is used to convert the signals. An estimation of the reduction of energy consumption in the refrigeration system due to the technology of cloud computing is given.
\end{abstract}

Keywords: Refrigeration Systems; Energy Efficiency; Cloud Computing; Intelligent Grids

\section{References}

1. Bera S., Misra S., Rodrigues P. C. Cloud Computing Applications for Smart Grid: A Survey 2017/ https://www.researchgate.net/publication/262116090

2. Qaisar E. Introduction to cloud computing for developers: Key concepts, the players and their offerings/ 2012. Proc. of IEEE TCF Pro IT, pp. 1-6.

3. Popeanga J. Cloud Computing and Smart Grids. 2012. Database Systems Journal, vol. 3, no. 3, pp. 57-66.

4. Lakhani A. The Definition of Cloud Computing. 2011. http://www.cloudcentrics.com

5. Mell P., and Grance T. The NIST Definition of Cloud Computing, US National Institute of Science and Technology Std., 2011. [Online]. Available: http://csrc.nist.gov/publications/nistpubs/800-145/SP800-

\section{5.pdf}

6. Guo Y., Pan M., and Fang Y. Optimal Power Management of Residential Customers in the Smart Grid,
2012. IEEE Trans. on Parallel and Distributed Systems, vol. 23, no. 9, pp. 1593 - 1606 .

7. Markovic D.S., et al. Smart power grid and cloud computing. 2013. Renew. Sustain. Energy Rev. 24, pp. $566-577$.

8. Nozaki Y., Tominaga T., Iwasaki N., and Takeuchi A. A technical approach to achieve smart grid advantages using energy management systems. 2011. Proc. of IEEE Intl. Conf. on WCSP, pp. 1-5.

9. Fang X., Misra S., and Yang D. Smart Grid. The New and Improved Power Grid: A Survey. 2012. IEEE Comm. Surveys \& Tutorials, vol. 14, no. 944-980.

10. Hashmi M., Hanninen S., and Maki K. Survey of smart grid concepts, architectures, and technological demonstrations worldwide. 2011. in Proc. of IEEE PES ISGT, pp. 1-7.

11. Zhou J., Hu R. Q., and. Qian Y. Scalable Distributed Communication Architectures to Support Advanced Metering Infrastructure in Smart Grid. 2012. IEEE Trans. 
on Parallel and Distributed Systems, vol. 23, no. 9, pp. 1632 - 1642 .

12. Metke A. and Ekl R. Smart Grid security technology. 2010. Proc. of IEEE Conf. on ISGT, pp. 1-7.

13. Luo F., Dong Z. Y., Chen Y., Xu Y., Meng K., and Wong K. P. Hybrid cloud computing platform: The next generation IT backbone for smart grid. 2012. Proc. of IEEE Conf. on PES General Meeting, pp. 1-7.

14. Metke A. and Ekl R. Smart Grid security technology. 2010. Proc. of IEEE Conf. on ISGT, pp. 1-7.

15. Smart Grids European Technology Platform. 2011. www.smartgrids.eu.

16. Goulden M., Redwell B., Rennick-Egglestone S., Rodden T., Spence A. 2014. Smart grids, smart users? The role of the user in demand side management Energy Research \& Social Science 2, 21-29

17. Petrenko M. O., Trishin F. A., Mazur V. O. (2017) Energy Efficient Refrigeration Systems in a Smart Grid. Refrigeration Engineering and Technology, 53 (4), 42-49 (in Russian) DOI: http://dx.doi.org/10.15673/ret.v53i4.708

18. Prodan R. and Ostermann S. A Survey and Taxonomy of Infrastructure as a Service and Web Hosting Cloud Providers. 2009. Proc. of Intl. Conf. on Grid Computing. 2009. pp. 1-10.
19. Asnafieva N.M. Veivlet analiz: osnovy teorii i primery primeneniia. 1996. Uspekhi fizicheskikh nauk. 66, No.11, 1146 - 1170 .

20. https://www.mathworks.com/products/wavelet.html

21 . Benefits of demand response in electricity markets and recommendations for achieving them. Report to the United States Congress, Feb. 2006. https://eetd.lbl.gov/sites/all/files/publications/report-lbnl-

\section{2d.pdf}

22. Erol-Kantarci M. and Mouftah H. TOU-Aware Energy Management and Wireless Sensor Networks for Reducing Peak Load in Smart Grids. , 2010. Proc. of IEEE Conf. on VTC-Fall, pp. 1-5.

23. Nezamabadi P. and. Gharehpetian G. Electrical energy management of virtual power plants in distribution networks with renewable energy resources and energy storage systems. 2011. Proc. of Electrical Power Distribution Networks, Bandar Abbas. pp. 1-5.

24. Mazur V. Fuzzy thermoeconomic optimization of energy-transforming systems. 2007. Applied Energy 84, pp. 749-762. 